\title{
ON A THEOREM OF REPRESENTATION OF LATTICES
}

\author{
S. P. WANG ${ }^{1}$
}

Let $G$ be a connected Lie group, $\Gamma$ a discrete subgroup of $G$ and $\mu$ a fixed right Haar measure of $G$. $\mu$ induces a measure $\bar{\mu}$ over $G / \Gamma$, the homogeneous space of left cosets of $\Gamma$. $\Gamma$ is called a lattice of $G$ if $\bar{\mu}(G / \Gamma)$ is finite. We denote the set of all representations of $\Gamma$ in $G$, i.e., all homomorphisms from $\Gamma$ into $G$ by $R(\Gamma, G)$. We shall give $R(\Gamma, G)$ the compact-open topology, Let $H$ be any closed subgroup of $G$. We use $U^{H}$ to mean the induced unitary representation of $G$ by the trivial one dimensional unitary representation of $H$. In this note, we are going to prove the following

Proposition. Let $G$ be a connected semisimple Lie group without compact factor, $\Gamma$ a lattice of $G$ and $\left\{r_{n}\right\}$ a sequence of representations of $\Gamma$ in $G$. If $\Gamma_{n}=r_{n}(\Gamma)$ are discrete for all $n$ and $\lim _{n} r_{n}=1_{\Gamma}$, then the trivial representation of $\Gamma$ is weakly contained in $\left\{\oplus U^{\Gamma_{n}} \mid \Gamma\right\} .^{2}$

As a consequence of the preceding proposition and results in [3] and [6], one can establish the following

THEOREM. Let $G$ be a connected semisimple Lie group with each factor of $R$-rank $\geqq 2, \Gamma$ a lattice of $G$ and $\left\{r_{n}\right\}$ a sequence of representations of $\Gamma$ in $G$. If $r_{n}(\Gamma)$ are all discrete and $\lim _{n} r_{n}=1_{\Gamma}$, then $r_{n}(\Gamma)$ are lattices of $G$ for sufficiently large values of $n$.

In general the theorem is not true without the restriction on the $\boldsymbol{R}$-rank of each factor of $G$. This can be illustrated by the classical counterexample in the case of the upper half-plane: the Hecke groups $\Gamma_{c}$ generated by

$$
\left(\begin{array}{rr}
0 & 1 \\
-1 & 0
\end{array}\right) \text { and }\left(\begin{array}{ll}
1 & c \\
0 & 1
\end{array}\right)
$$

for $c \geqq 2$. They are all isomorphic to $\Gamma_{2}$, but only $\Gamma_{2}$ is a lattice.

Before we give the proofs of the proposition and the theorem, let us verify some lemmas needed later.

Lemma 1. Let $G$ be a semisimple Lie group without compact factor, $\Gamma$ a lattice of $G$ and $\left\{r_{n}\right\}$ a sequence of representations of $\Gamma$ in $G$ such that $\Gamma_{n}=r_{n}(\Gamma)$ are all discrete. If $\lim _{n} r_{n}=1_{\Gamma}$, then we have

Received by the editors November 9, 1968.

1 The author would like to thank the referee for helpful suggestions.

${ }^{2}$ For weak containment, see [2]. 
(i) $\left\{\Gamma_{n}\right\}$ is uniformly discrete, i.e., there exists a neighborhood $V$ of identity $e$ in $G$ with $\Gamma_{n} \cap V=\{e\}$ for all $n$.

(ii) $\Gamma \cap Z(G)$ is of finite index in $Z(G)$ and $\left.r_{n}\right|_{\Gamma \cap Z(G)}=1_{\Gamma \cap Z(G)}$ for sufficiently large value of $n$, where $Z(G)$ is the center of $G$.

Proof. Since $\Gamma$ is a lattice, by the density theorem of lattices [1], $Z(G) \Gamma$ is also a lattice. Hence it follows easily that $\Gamma \cap Z(G)$ is of finite index in $Z(G)$. Let $\hat{G}$ be the Lie algebra of $G$ and Ad: $G \rightarrow G L(\hat{G})$ the adjoint representation of $G$. By the density theorem $[1], l(\operatorname{Ad}(\Gamma))$, the linear span of $\operatorname{Ad}(\Gamma)$ in $\operatorname{End}(\hat{G})$, equals $l(\operatorname{Ad}(G))$. Since $\lim _{n} r_{n}$ $=1_{\mathrm{r}}, \operatorname{dim} l\left(\operatorname{Ad}\left(\Gamma_{n}\right)\right)=\operatorname{dim} l(\operatorname{Ad}(\Gamma))=\operatorname{dim} l(\operatorname{Ad}(G))$ for sufficiently large $n$. Therefore $l\left(\operatorname{Ad}\left(\Gamma_{n}\right)\right)=l(\operatorname{Ad}(G))$ holds for large $n$. It follows in particular that the $r_{n}(\Gamma \cap Z(G))$ are central for large $n$. As $Z(G)$ is discrete and $\Gamma \cap Z(G)$ finitely generated, $r_{n} \mid \Gamma \cap_{Z(G)}=1_{\Gamma \cap Z(G)}$ for sufficiently large $n$.

Lemma 2. Let $G$ be a Lie group, $\mu$ a Haar measure, and $U, V, W$ three $\mu$-measurable subsets of $G$. If $\mu(V-W)+\mu(W-V)<\epsilon$, then

$$
|\mu(U-V)-\mu(U-W)|<\epsilon .
$$

Proof. $\quad U-V=(U-(V \cup W)) \cup((W-V) \cap U)$ and $\quad U-W$ $=(U-(V \cup W)) \cup((V-W) \cap U)$.

Hence

$$
|\mu(U-V)-\mu(U-W)| \leqq \mu(V-W)+\mu(W-V)<\epsilon .
$$

COROLlaRy 3. Let $U$ and $V$ be two finite $\mu$-measurable open subsets of $G$ and $\left(\gamma_{1}^{(m)}, \ldots, \gamma_{n}^{(m)}\right) \in G^{n}$ such that $\lim _{m}\left(\gamma_{1}^{(m)}, \cdots, \quad \gamma_{n}^{(m)}\right)=$ $\left(\gamma_{1}, \cdots, \gamma_{n}\right) \in G^{n}$.

Then

$$
\lim _{m} \mu\left(U-V \gamma_{1}^{(m)}-\cdots-V \gamma_{n}^{(m)}\right)=\mu\left(U-V \gamma_{1}-\cdots-V \gamma_{n}\right) .
$$

Proof. Let $W$ be any finite $\mu$-measurable open subset of $G$. Then the function $f_{W}(x)=\mu(W-W x), x \in G$ is continuous. Hence

$$
\underset{m}{\operatorname{iim}} \mu\left(V \gamma_{i}-V \gamma_{i}^{(m)}\right)=0 \text { and } \lim _{m} \mu\left(V \gamma_{i}^{(m)}-V \gamma_{i}\right)=0,
$$

for $1 \leqq i \leqq n$. Then by Lemma 2 , the corollary follows immediately.

Corollary 4. Let $U$ and $V$ be two finite $\mu$-measurable open subsets, $\Gamma$ a discrete subgroup of $G$ and $\left\{r_{n}\right\}$ a sequence of representations of $\Gamma$ in $G$ such that $\lim _{n} r_{n}=1_{\Gamma}$. Then $\lim _{n}$ sup $\mu\left(U-V T_{n}\right) \leqq \mu(U-V T)$ where $\Gamma_{n}=r_{n}(\Gamma)$ for all $n$. 
Proof. As $\Gamma$ is a discrete subgroup of $G, \Gamma$ is countable. Let $\Gamma=\left\{\gamma_{1}, \cdots, \gamma_{i}, \cdots\right\}$ and $U_{n}=U-V \gamma_{1}-\cdots-V \gamma_{n}$. Then $\left\{U_{n}\right\}$ is a decreasing sequence of finite $\mu$-measurable subsets of $G$. Since $\cap_{n} U_{n}=U-V \Gamma, \lim _{n} \mu\left(U_{n}\right)=\mu(U-V \Gamma)$. Let $\epsilon$ be any positive number. There exists a positive integer $N$ such that $0 \leqq \mu\left(U_{n}\right)$ $-\mu(U-V \Gamma)<\epsilon$ for all $n \geqq N$. Let $\gamma_{i}^{(m)}=r_{m}\left(\gamma_{i}\right), i=1,2, \cdots$. Clearly $\lim _{m}\left(\gamma_{1}^{(m)}, \cdots, \gamma_{n}^{(m)}\right)=\left(\gamma_{1}, \cdots, \gamma_{n}\right)$. Thus by Corollary 3 ,

$$
\lim _{m} \mu\left(U-V \gamma_{1}^{(m)}-\cdots-V \gamma_{N}^{(m)}\right)=\mu\left(l_{N}\right)<\mu(U-V \Gamma)+\epsilon .
$$

Since $\mu\left(U-V \Gamma_{m}\right) \leqq \mu\left(-V \gamma_{1}^{(m)}-\cdots-V \gamma_{N}^{(m)}\right)$ for all $m, \lim _{m}$ sup $\mu\left(U-V \Gamma_{m}\right) \leqq \mu(U-V \Gamma)+\epsilon$. However $\epsilon$ is an arbitrary positive number. This implies

$$
\lim _{n} \sup \mu\left(U-V \Gamma_{n}\right) \leqq \mu(U-V \Gamma) .
$$

Now we are ready to prove the proposition and the theorem.

Proof of THE PROPOSITION. By Lemma 1, there exists an open neighborhood $W$ of $e$ in $G$ such that $W^{-1} W \cap \Gamma_{n}=\{e\}$ for all $n$. By constructing it properly, we can find a fundamental domain $F$ of $\Gamma$ such that $W \subset F$ and the boundary of $F$ has $0 \mu$-measure. Let $V$ be the interior of $F$. It is clear that $\mu(V)=\bar{\mu}(G / \Gamma)<\infty$ and $\mu(x V-V \Gamma)=0$ for all $x \in G$. Therefore by Corollary $4, \lim _{n} \mu\left(x V-V \Gamma_{n}\right)=0$. Let $\bar{\mu}_{n}$ be the invariant measure of $G / \Gamma_{n}$ induced from $\mu$. Then we have

$$
\begin{aligned}
\bar{\mu}_{n}\left(x V \Gamma_{n}-V \Gamma_{n}\right) & =\bar{\mu}_{n}\left(\left(x V-V \Gamma_{n}\right) \Gamma_{n}\right) \\
& \leqq \mu\left(x V-V \Gamma_{n}\right), \quad \text { for all } n,
\end{aligned}
$$

and all $x \in G$. Hence $\lim _{n} \bar{\mu}_{n}\left(x V \Gamma_{n}-V \Gamma_{n}\right)=0$. Since $W^{-1} W \cap \Gamma_{n}=\{e\}$ and $W \subset V, \bar{\mu}_{n}\left(V \Gamma_{n}\right) \geqq \mu(W)$. Now let $\left\{\gamma_{1}, \cdots, \gamma_{m}\right\}$ be any finite subset of $\Gamma$. We have $\lim _{n} \bar{\mu}_{n}\left(\gamma_{i} V \Gamma_{n}-V \Gamma_{n}\right) / \bar{\mu}_{n}\left(V \Gamma_{n}\right)=0,1 \leqq i \leqq \mathrm{~m}$. Now denote the characteristic function of $V \Gamma_{n}$ in $G / \Gamma_{n}$ by $\chi_{n}$, i.e., $\chi_{n}(g \Gamma)=1$ or 0 according as $g \in V \Gamma_{n}$ or not, and $f_{n}=\bar{\mu}_{n}\left(V \Gamma_{n}\right)^{-1 / 2} \chi_{n}$. By the definition of induced representation, $f_{n}$ is in the Hilbert space of the unitary representation $U^{\Gamma_{n}}$. Due to our construction, $\lim _{n}\left\langle\gamma_{i} f_{n}, f_{n}\right\rangle=1$, $1 \leqq i \leqq m$. Therefore the trivial representation of $\Gamma$ is weakly contained in $\left\{\oplus U^{\Gamma_{n}} \mid \Gamma\right\}$.

Proof of THE THEOREM. We shall prove it first for the case when $G$ has no nontrivial center. Suppose the theorem to be false in this case. Then there is a subsequence $\left(n^{\prime}\right)$ of $(n)$ such that $\Gamma_{n^{\prime}}$ are not lattices of $G$ for all $n^{\prime}$. By the proposition, the trivial representation $I$ of $\Gamma$ is weakly contained in $\left\{\oplus U^{\Gamma^{\prime}} \mid \Gamma\right\}$. However $\Gamma$ has property $(T)$ of [3] and [6]. It follows that $I$ is a subrepresentation of $\oplus U^{\Gamma_{n}} \mid \Gamma$. Therefore $I \leqq U^{\Gamma_{n}} \mid \Gamma$ for certain $n^{\prime}$, say $n_{0}^{\prime}$. Then by Lemma 
3.4 in [6], $U^{\Gamma_{n}{ }_{0}}$ contains the trivial representation of $G$. By Lemma 8.2 in [4], $\Gamma_{n^{\prime} 0}$ has to be a lattice of $G$, which contradicts our choice of $\left(n^{\prime}\right)$. Next we shall reduce the general case to the previous one. Let $Z(G)$ be the center of $G$. By Lemma 1 (ii), $Z(G) \cap \Gamma$ is a subgroup of finite index in $Z(G)$ and $r_{n} \mid Z(G) \cap \Gamma=1 Z(G) \cap \Gamma$ for sufficiently large $n$. Denote $G / Z(G), \Gamma Z(G) / Z(G), \Gamma_{n} Z(G) / Z(G)$ by $\bar{G}, \bar{\Gamma}$ and $\bar{\Gamma}_{n}$ respectively. Clearly for large $n, r_{n}$ induce $r_{n}: \bar{\Gamma} \rightarrow \bar{G}$ such that $r_{n}(\bar{\Gamma})=\bar{\Gamma}_{n}$. Since $r_{n \mid Z(G) \cap \Gamma}=1_{Z(G) \cap \Gamma}$ for large $n$ and $Z(G) \cap \Gamma$ is of finite index in $Z(G)$, the $\bar{\Gamma}_{n}$ are discrete for large $n$. Therefore $\bar{G},\left\{\bar{r}_{n}\right\}$, and $\bar{\Gamma}_{n}$ satisfy the conditions of the theorem and $\bar{G}$ has no nontrivial center. By what we have just proved, the $\bar{\Gamma}_{n}$ are lattices of $\bar{G}$ for large $n$. Since $\Gamma_{n}$ contains $Z(G) \cap \Gamma$, which is of finite index in $Z(G)$, for large $n$, one concludes readily that the $\Gamma_{n}$ are lattices of $G$ for sufficiently large $n$.

As suggested by the referee, the proposition and theorem can be generalized slightly in the following case. $\Gamma$ is a finitely generated group, $r_{0}: \Gamma \rightarrow G$ a homomorphism whose image is a lattice, and $r_{n}$ : $\Gamma_{n} \rightarrow G$ homomorphisms whose images are discrete, and which tend to $r_{0}$. It is easy to see that the slightly more general form can be obtained from the following lemma and our results.

Lemma 5. Let $\mathrm{G}$ be a connected Lie group, $\Gamma$ a finitely generated group, $r_{0}: \Gamma \rightarrow G$ a homomorphism whose image is discrete, and $r_{n}: \Gamma \rightarrow G$ homomorphisms whose images are uniformly discrete and which tend to $r_{0}$. Then there exist $r_{n}^{\prime} \in R\left(r_{0}(\Gamma), G\right)$ such that $r_{n}=r_{n}^{\prime} \cdot r_{0}$ for large $n$ and $\lim _{n} r_{n}^{\prime}=1_{r_{0}(\Gamma)}$.

Proof. Let $\left\{\gamma_{1}, \cdots, \gamma_{2 m}\right\}$ be a set of generators of $\Gamma$ such that $\gamma_{i}^{-1}=\gamma_{i+m}, 1 \leqq i \leqq m$. Denote $r_{i}\left(\gamma_{j}\right)$ by $\gamma_{j}^{(i)}, 1 \leqq j \leqq 2 m, i=1,2, \cdots$. By the same argument as used in Theorem 4 [7], we know that there are finitely many relations $R_{1}, \cdots, R_{N}$ satisfied by $\left(\gamma_{1}^{(0)}, \cdots, \gamma_{2 m}^{(0)}\right)$ such that for any $\left(x_{1}, \cdots, x_{2 m}\right) \in G^{2 m}$ satisfying $R_{1}, \cdots, R_{n}$ and close enough to $\left(\gamma_{1}^{(0)}, \cdots, \gamma_{2 m}^{(0)}\right)$, the map $\gamma_{j}^{(0)} \rightarrow x_{j}(1 \leqq j \leqq 2 m)$ extends to a homomorphism of $r_{0}(\Gamma)$ into $G$. Since the $r_{n}(\Gamma)$ are uniformly discrete and $\lim _{i} \gamma_{j}^{(i)}=\gamma_{j}^{(0)}, 1 \leqq j \leqq 2 m$, one sees easily that $\gamma_{j}^{(0)} \rightarrow \gamma_{j}^{(i)}, 1 \leqq j \leqq 2 m$ extend to homomorphisms $r_{i}^{\prime}: r_{0}(\Gamma) \rightarrow G$ for large $i$. Now the lemma follows immediately.

\section{BibliogRAPHY}

1. A. Borel, Density properties of certain subgroups of semisimple groups without compact factor, Ann. of Math. (2) 72 (1960), 179-188.

2. J. M. G. Fell, Weak containment and induced representation of groups, Canad. J. Math. 14 (1962), 237-268.

3. D. A. Kazhdan, Connection of the dual space of a group with the structure of its closed subgroups, Funkcional. Anal. i Priložen 1 (1967), 71-74. 
4. G. W. Mackey, Induced representation of locally compact groups, I. Ann. of Math. (2) 55 (1962), 101-139.

5. H. C. Wang, On a maximality property of discrete subgroup, with fundamental domain of finite measure, Amer. J. Math. 89 (1967), 124-132.

6. S. P. Wang, The dual space of semi-simple Lie groups, Amer. J. Math. (to appear).

7. - Limit of lattices in a Lie Group, Trans. Amer. Math. Soc. 133 (1968), 519-526.

Institute for Advanced Study 